\title{
Ethnobotanical Survey on Wild Edible Plants Used by Tribals \& Rural People of Arjuni/Mor Taluka, Gondia District, Maharashtra State, India
}

\author{
Kailash S. Lokhande \\ Department of Botany, S.S.Jaiswal College, India \\ Received February 22, 2020; Revised March 20, 2020; Accepted March 28, 2020
}

Copyright $\odot 2020$ by authors, all rights reserved. Authors agree that this article remains permanently open access under the terms of the Creative Commons Attribution License 4.0 International License

\begin{abstract}
The study area is inhabited by Gond tribes. Tribals and most of forest dwellers mainly depend on plants and plant product for their food. This study reveals that the ethnic people and forest dwellers have considerable traditional knowledge of wild edible plants and their utilization. This paper report is based on survey, interview and field work studies on wild edible plants used by tribals and rural people of Arjuni/Mor Taluka, Gondia District, Maharashtra State, India during March 2016 - March 2018. A total of 71 plant species belonging to 63 genera and 39 families have been recorded as wild edibles in the study areas, of which trees are ranked first with 36 species, followed by herbs, climbers and shrubs with 22, 7 and 6 species respectively. Among the 71 species, 43.2\% contribute as vegetables by their fruit, $22.22 \%$ by leaf, $12.34 \%$ by seed or grain, $8.64 \%$ by root or tuber, $6.17 \%$ by stem, bark or sap, $6.17 \%$ by flower and $1.23 \%$ by whole plant. It is evident from the survey that most of the species of wild edibles are used as vegetable/chutney(49.33\%), followed by raw/ripe fruits(45.33\%), pickles(4\%), roasted seeds $(1.33 \%)$.
\end{abstract}

Keywords Ethnic People, Traditional Knowledge, Forest Dwellers, Commercial Food

\section{Introduction}

Ethnobotany encompasses the total, natural and traditional man-plant relationships. It recognizes the important role of the ambient vegetation in the economic life of people. Ethnobotany has now contributions to an understanding of man-plant relationships, as well as for the practical applications of the biological knowledge of aboriginal people in medicine, health, agriculture and industry. [1]

From ancient time plants have been used as a source of food, shelter, clothing, medicine, fibre, gum, resin, oil, etc. Several wild plants are used as food by tribals and other local people living in and around the forest areas. [2]

Tribal and local people prefer wild vegetables over the cultivated as they grow naturally; provide better taste and good health. By selling these wild edibles to the nearby urban markets could earn them extra income. Aboriginals believed that some of the seasonal wild vegetables are good for health and also provide the immunity during the rains which is supposed to be the most suspicious period to become ill by various disorders. [3]

Now the ancient men are thoroughly acquainted with the method of excluding the harmful substances from the wild plants and preparing recipes for their meager meals. [4]

Food plants are not only supplement to the food quantity, but also an important option during starvation for survival and thus makes significant contribution to the human nutrition throughout the year. However, the century old traditional knowledge system for utilization of wild plants is depleting very quickly. Modern scientific researchers are trying to value these traditional food items to fill the gaps between growing population and food production. [5]

The present studies were conducted to explore the knowledge of wild edible plants to meet the increasing demands of the growing population. The diversity of these plant resources needs to be documented and analysis should be done for their nutritional values. Increased use of traditional vegetables can contribute to enhancing people's health and standard living as well as the economic and social status of the food producers themselves.

\section{Materials and Methods}

\subsection{Study Area}

This study was conducted in 11 villages in Arjuni/Mor 
Taluka of Gondia district, Maharashtra state, India. Gondia district is situated between $20^{\circ} 39^{\prime}$ to $21^{\circ} 38^{\prime} \mathrm{N}$ and $79^{\circ} 27^{\prime}$ to $80^{\circ} 42^{\prime} \mathrm{E}$ (Fig.1). The district is located in the north-eastern part of the state and is bordered by the states of Chhattisgarh and Madhya Pradesh from east and north respectively and Bhandara district and Chandrapur district of Maharashtra to the west and south.

Gondia district was under the privilege of 'Gond Raja'. The whole area was surrounded by the dense forest. 'Gond ' is the main tribe of this area. [6]

Surban, Bondgaon, Gothangaon, Pratapgad, Navegaon, Dewalgaon, Khairi, Sukadi, Dabhana, Tidka, Kesori are the villages in study area. All these 11 villages are surrounded by dense forest.
Study was carried out during the year of March 2016 to March 2018. This Information on wild edible plant species is outcome of ethnobotanical field survey and interviews among old tribal peoples, ethnic men and women based on the standard procedures suggested by. [7] The field survey covered different seasons. Data collection was made in different places i.e. forest, bare lands, playground, roadside, agricultural farms and near localities. Plant specimens were collected and identified with the help of floras. [8-12]

Notes were recorded on local names, useful plant parts and their utilization by showing photographs of some collected specimens to local peoples. The identified plants are arranged alphabetically with family names, local names and parts used (Table 1).

\subsection{Data Collection}

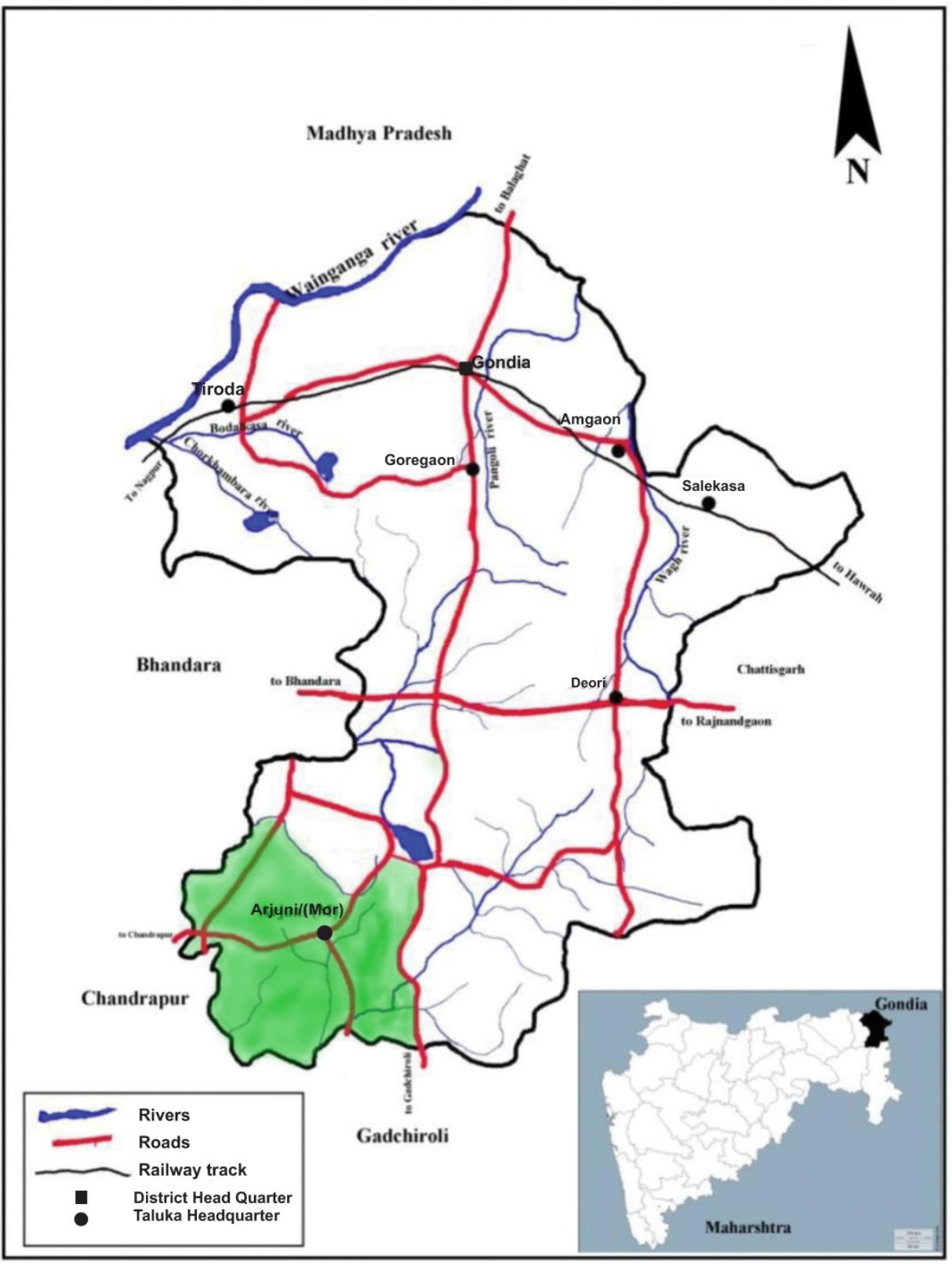

Figure 1. Showing Study Area in Gondia District Map 
Table 1. Wild edible plants used by Local people of study area

\begin{tabular}{|c|c|c|c|c|c|c|}
\hline S.N & Plant Name & Family & Local Name & $\begin{array}{l}\text { Life } \\
\text { form }\end{array}$ & Part(s) used & Mode of utilization \\
\hline 1. & $\begin{array}{l}\text { Abelmoschus moschatus } \\
\text { Medic. }\end{array}$ & Malvaceae & Kasthoori bhendi & $\mathrm{H}$ & Fruits & Cooked as vegetable \\
\hline 2. & Abrus precatorius $\mathrm{L}$. & Fabaceae & Gomchi, Gunja & $\mathrm{C}$ & Leaves & Used as vegetable \\
\hline 3. & Abutilon indicum (L.) Sweet & Malvaceae & Petari & $\mathrm{H}$ & $\begin{array}{l}\text { Leaves and } \\
\text { Flowers }\end{array}$ & Cooked as vegetable \\
\hline 4. & Acacia catechu (L.f.) Willd & Mimosaceae & Khair & $\mathrm{T}$ & Gum & Taken with betel leaf \\
\hline 5. & Acacia nilotica (L.) Willd. & Mimosaceae & Babul & $\mathrm{T}$ & Gum and seeds & $\begin{array}{l}\text { Seeds roasted with } \\
\text { salt and eaten }\end{array}$ \\
\hline 6. & Aegle marmelos Corr. & Rutaceae & Bel & $\mathrm{T}$ & Fruit & $\begin{array}{l}\text { Raw fruit pulp is } \\
\text { eaten }\end{array}$ \\
\hline 7. & Alternanthera sessilis DC & Amaranthaceae & Jibhkati & $\mathrm{H}$ & Leaves & Cooked as vegetable \\
\hline 8. & Amaranthus spinosus $\mathrm{L}$. & Amaranthaceae & Matbhaji & $\mathrm{H}$ & Leaves & Cooked as vegetable \\
\hline 9. & Amaranthus viridis $\mathrm{L}$. & Amaranthaceae & Khedabhaji & $\mathrm{H}$ & Leaves & Cooked as vegetable \\
\hline 10. & $\begin{array}{c}\text { Amorphophalus paeonifolius } \\
\text { Dernst. }\end{array}$ & Araceae & Jimikanda & $\mathrm{H}$ & $\begin{array}{l}\text { Tender Leaves } \\
\text { and Corm }\end{array}$ & Cooked as vegetable \\
\hline 11. & Annona reticulate $\mathrm{L}$. & Annonaceae & Ramphal & $\mathrm{T}$ & Ripe fruits & Eaten raw \\
\hline 12. & Annona squamosa $\mathrm{L}$. & Annonaceae & Sitaphal & $\mathrm{T}$ & Ripe fruits & Eaten raw \\
\hline 13. & $\begin{array}{c}\text { Artocarpus hetrophyllus } \\
\text { Lamk }\end{array}$ & Moraceae & Phanas & $\mathrm{T}$ & Fruits & Cooked as vegetable \\
\hline 14. & $\begin{array}{c}\text { Antidesma ghaesembilla } \\
\text { Gaertn }\end{array}$ & Euphorbiaceae & Amti,Jondhurli & $\mathrm{T}$ & Ripe fruits & Eaten raw \\
\hline 15. & Averrhoa carambola $\mathrm{L}$. & Oxalidaceae & Aamras & $\mathrm{T}$ & Ripe fruits & Eaten raw \\
\hline 16. & Azadirachta indica A.Juss. & Meliaceae & Nim & $\mathrm{T}$ & Ripe fruits & Eaten raw \\
\hline 17. & Bacopa monnieri (L.) Penn. & Scrophulariaceae & Brahmi & $\mathrm{T}$ & Whole plant & Cooked as vegetable \\
\hline 18. & Bambusa vulgaris Schrad, & Poaceae & Bans, Vadud & $\mathrm{T}$ & Tender Shoots & Cooked as vegetable \\
\hline 19. & Bauhinia purpurea L. & Caesalpiniaceae & Koilar & $\mathrm{T}$ & $\begin{array}{l}\text { Leaves and } \\
\text { Flower buds }\end{array}$ & Cooked as vegetable \\
\hline 20. & Bauhinia vahlii Wight \& Arn. & Caesalpiniaceae & Mahulbel & $\mathrm{C}$ & Seeds & Cooked as vegetable \\
\hline 21. & Bridelia retusa Spreng & Euphorbiaceae & Kasai & $\mathrm{T}$ & Ripe fruits & Eaten raw \\
\hline 22. & $\begin{array}{l}\text { Buchanania cochinchinesis } \\
\text { (Lour.) Almeida }\end{array}$ & Anacardiaceae & Sadeka, Char & $\mathrm{T}$ & $\begin{array}{l}\text { Ripe Fruits and } \\
\text { Seeds }\end{array}$ & Eaten raw \\
\hline 23. & $\begin{array}{l}\text { Butea monosperma (Lam.) } \\
\text { Taub. }\end{array}$ & Fabaceae & Parsa, Palas & $\mathrm{T}$ & Flower & Cooked as vegetable \\
\hline 24. & $\begin{array}{c}\text { Cajanus scarabaeoides (L.) } \\
\text { Du Petit- thou }\end{array}$ & Fabaceae & Ran- tur & $\mathrm{H}$ & Seeds & Cooked as vegetable \\
\hline 25. & $\begin{array}{c}\text { Cardiospermum helicacabum } \\
\text { L. }\end{array}$ & Sapindaceae & Kaparphuti & $\mathrm{H}$ & Ripe fruits & Eaten raw \\
\hline 26. & Cassia fistula $\mathrm{L}$. & Caesalpiniaceae & Bahava & $\mathrm{T}$ & $\begin{array}{c}\text { Flowers and } \\
\text { seeds }\end{array}$ & Cooked as vegetable \\
\hline 27. & Cassia tora $\mathrm{L}$. & Caesalpiniaceae & Charota, Chirota & $\mathrm{H}$ & Leaves & Cooked as vegetable \\
\hline 28. & Chenopodium album $\mathrm{L}$. & Chenopodiaceae & Awadi-dhawadi & $\mathrm{H}$ & Leaves & Cooked as vegetable \\
\hline 29. & Cissus quadrangularis $\mathrm{L}$. & Vitaceae & Hadijod & $\mathrm{C}$ & Shoot & Cooked as vegetable \\
\hline 30. & $\begin{array}{c}\text { Colocasia esculenta (L.) } \\
\text { Schott }\end{array}$ & Araceae & $\begin{array}{l}\text { Dhopa,Aaki, } \\
\text { Kochaimati, }\end{array}$ & $\mathrm{H}$ & $\begin{array}{l}\text { Leaves, petiole } \\
\quad \& \text { tuber }\end{array}$ & Cooked as vegetable \\
\hline 31. & Commelina benghalensis $\mathrm{L}$. & Commelinaceae & Telka bhaji & $\mathrm{H}$ & Leaves & Cooked as vegetable \\
\hline 32. & Costus speciosus (Koen) Sm. & Zingiberaceae & Kevkanda & $\mathrm{H}$ & Leaves, Tuber & Cooked as vegetable \\
\hline 33. & $\begin{array}{l}\text { Cryptocoryne retrospiralis } \\
\text { (Roxb.) Kunth }\end{array}$ & Araceae & Pakanbhed & $\mathrm{H}$ & Leaves & Cooked as vegetable \\
\hline 34. & $\begin{array}{c}\text { Dendrocalamus strictus } \\
\text { (Roxb.) Nees }\end{array}$ & Poaceae & Ranj, bamboo & $\mathrm{T}$ & Tender shoots & Cooked as vegetable \\
\hline 35. & Dioscorea bulbifera L. & Dioscoreaceae & Mataru, Dangkanda & $\mathrm{C}$ & Tuber, bulbils & Cooked as vegetable \\
\hline 36. & Diospyros melanoxylon Roxb. & Ebenaceae & Tembhrun, Tendu & $\mathrm{T}$ & Ripe Fruits & Eaten raw \\
\hline 37. & Ficus racemosa $\mathrm{L}$. & Moraceae & Umbar & $\mathrm{T}$ & Ripe Fruits & Eaten raw \\
\hline
\end{tabular}


Table 1 Continued

\begin{tabular}{|c|c|c|c|c|c|c|}
\hline 38. & Gardenia lattifolia Ait. & Rubiaceae & Ghogar & $\mathrm{T}$ & Ripe Fruits & Eaten raw \\
\hline 39. & Gmelina arborea Roxb & Verbenaceae & Siwan & $\mathrm{T}$ & Ripe Fruits & Eaten raw \\
\hline 40. & Grewia hirsuta Vahl. & Tiliaceae & Gaturli/Gautri & $\mathrm{S}$ & Ripe Fruits & Eaten raw \\
\hline 41. & Grewia tiliifolia Vahl Fruits & Tiliaceae & Dhaman & $\mathrm{T}$ & Ripe Fruits & Eaten raw \\
\hline 42. & Hemidesmus indicus (L.) R. Br. & Periplocaceae & Khaprilbela & $\mathrm{C}$ & Tuber & Cooked as vegetable \\
\hline 43. & $\begin{array}{c}\text { Holarrhena pubescens } \\
\text { (Buch-Ham.)Wall.ex. G.Don. }\end{array}$ & Apocynaceae & Kudva & $\mathrm{S}$ & Flower, Fruits & Cooked as vegetable \\
\hline 44. & Ipomoea aquatica Lour & Convolvulaceae & Karmotabhaji & $\mathrm{H}$ & Leaves & Cooked as vegetable \\
\hline 45. & Lantana camara $\mathrm{L}$. & Verbenaceae & Bantulsi & $\mathrm{S}$ & Ripe Fruits & Eaten raw \\
\hline 46. & Limonia acidissima $\mathrm{L}$. & Rutaceae & Kawath & $\mathrm{T}$ & Ripe Fruits & $\begin{array}{l}\text { Eaten raw/used in } \\
\text { making pickles }\end{array}$ \\
\hline 47. & $\begin{array}{l}\text { Madhuca longifolia (Koen.) } \\
\text { Mac. Var. }\end{array}$ & Sapotaceae & Mahuva,Mahu & $\mathrm{T}$ & $\begin{array}{l}\text { Ripe Fruits, } \\
\text { Petals }\end{array}$ & $\begin{array}{l}\text { Eaten raw/ Cooked } \\
\text { as vegetable }\end{array}$ \\
\hline 48. & Mangifera indica $\mathrm{L}$. & Anacardiaceae & Aamba & $\mathrm{T}$ & Ripe Fruits & $\begin{array}{l}\text { Eaten raw/used in } \\
\text { making pickles }\end{array}$ \\
\hline 49. & Mimusops elengi $\mathrm{L}$. & Sapotaceae & Massor & $\mathrm{T}$ & Ripe Fruits & Eaten raw \\
\hline 50. & $\begin{array}{l}\text { Momordica dioica Roxb.ex } \\
\text { Willd. }\end{array}$ & Cucurbitaceae & Katwell & $\mathrm{C}$ & Fruits & Cooked as vegetable \\
\hline 51. & Moringa oleifera Lam. & Moringaceae & Mugna & $\mathrm{T}$ & Fruits & Eaten raw \\
\hline 52. & Mucuna pruriens (L.) DC & Fabaceae & Kanjkuri,Kavaskuri & $\mathrm{C}$ & Seeds & Cooked as vegetable \\
\hline 53. & Nelumbo nucifera Gaertn. & Nelumbonaceae & Kamal, Pavan & $\mathrm{H}$ & $\begin{array}{c}\text { Seeds, } \\
\text { Rhizomes }\end{array}$ & $\begin{array}{c}\text { Eaten raw/ Cooked } \\
\text { as vegetable }\end{array}$ \\
\hline 54. & Nymphaea nouchali Burm. f. & Nymphaeaceae & Kamal & $\mathrm{H}$ & $\begin{array}{l}\text { Peduncles, } \\
\text { Rhizomes }\end{array}$ & Cooked as vegetable \\
\hline 55. & Nymphaea rubra Roxb. & Nymphaeaceae & Lalkamal & $\mathrm{H}$ & Rhizomes & Cooked as vegetable \\
\hline 56. & Olax psittacorum (Willd) Vahl & Olacaceae & Aradphari/Harduli & $\mathrm{S}$ & Leaves & Cooked as vegetable \\
\hline 57. & Oryza rufipogon Griff. & Poaceae & Devdhan & $\mathrm{H}$ & Ripe fruits & Cooked as food \\
\hline 58. & Oxalis corniculata $\mathrm{L}$ & Oxalidaceae & Khatti Bhaji & $\mathrm{H}$ & Leaves & Cooked as vegetable \\
\hline 59. & Phoenix sylvestris (L.) Roxb. & Araceae & Sindi & $\mathrm{T}$ & Ripe fruits & Eaten raw \\
\hline 60. & Phyllanthus emblica $\mathrm{L}$. & Euphorbiaceae & Amla & $\mathrm{T}$ & Ripe fruits & $\begin{array}{l}\text { Eaten raw/used in } \\
\text { making pickles }\end{array}$ \\
\hline 61. & Physalis minima $\mathrm{L}$. & Solanaceae & Chirpoti & $\mathrm{H}$ & Ripe fruits & Eaten raw \\
\hline 62. & $\begin{array}{l}\text { Pithecellobium dulce (Roxb.) } \\
\text { Benth. }\end{array}$ & Mimosaceae & Chichbilai & $\mathrm{T}$ & Seeds & Eaten raw \\
\hline 63. & $\begin{array}{c}\text { Schleichera oleosa (lour.) O. } \\
\text { Ken }\end{array}$ & Sapindaceae & Kusum & $\mathrm{T}$ & Seeds & Eaten raw \\
\hline 64. & Semecarpus anacardium L.f. & Anacardiaceae & Biba/Bhilawa & $\mathrm{T}$ & Thalamus & Eaten raw \\
\hline 65. & Solanum nigrum $\mathrm{L}$. & Solanaceae & Kamuni & $\mathrm{S}$ & Leaves, fruits & $\begin{array}{c}\text { Fruits eaten raw and } \\
\text { leaves cooked as } \\
\text { vegetable. }\end{array}$ \\
\hline 66. & Syzygium cumini (L.) Skeels & Myrtaceae & Jambhul & $\mathrm{T}$ & Ripe fruits & Eaten raw \\
\hline 67. & Tamarindus indica. $\mathrm{L}$ & Caesalpiniaceae & Chich, Imli & $\mathrm{T}$ & Leaves, fruits & $\begin{array}{c}\text { Fruits eaten raw and } \\
\text { leaves cooked as } \\
\text { vegetable. }\end{array}$ \\
\hline 68. & Terminalia bellirica Roxb. & Combretaceae & Behda & $\mathrm{T}$ & Seeds & Eaten raw \\
\hline 69. & $\begin{array}{c}\text { Trapa natans L. var. bispinosa } \\
\text { (Roxb.) } \\
\text { Makino } \\
\end{array}$ & Trapaceae & Shingara & $\mathrm{H}$ & Fruits & Fruits eaten boiled \\
\hline 70. & Ziziphus mauritiana Lam. & Rhamnaceae & Boir, Ber & $\mathrm{T}$ & Ripe fruits & Eaten raw \\
\hline 71. & Ziziphus oenoplia (L.) Mill. & Rhamnaceae & Ironi & $\mathrm{S}$ & Ripe fruits & Eaten raw \\
\hline
\end{tabular}

H- Herbs; C-Climbers; S-Shrubs; T-Trees 


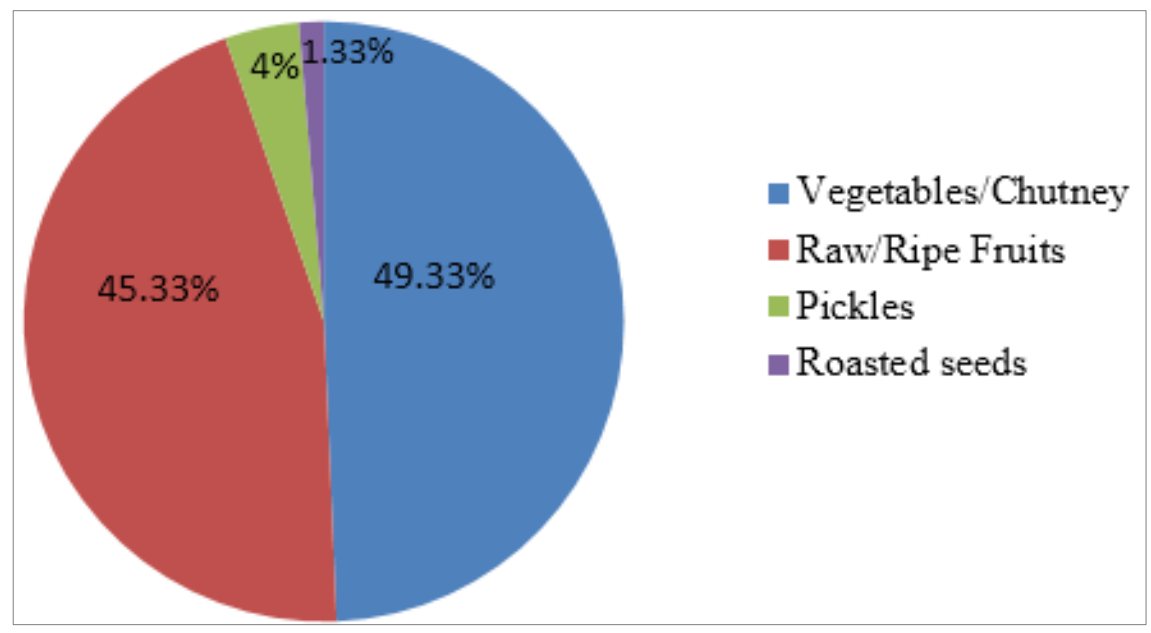

Figure 2. Classification on the basis of usage

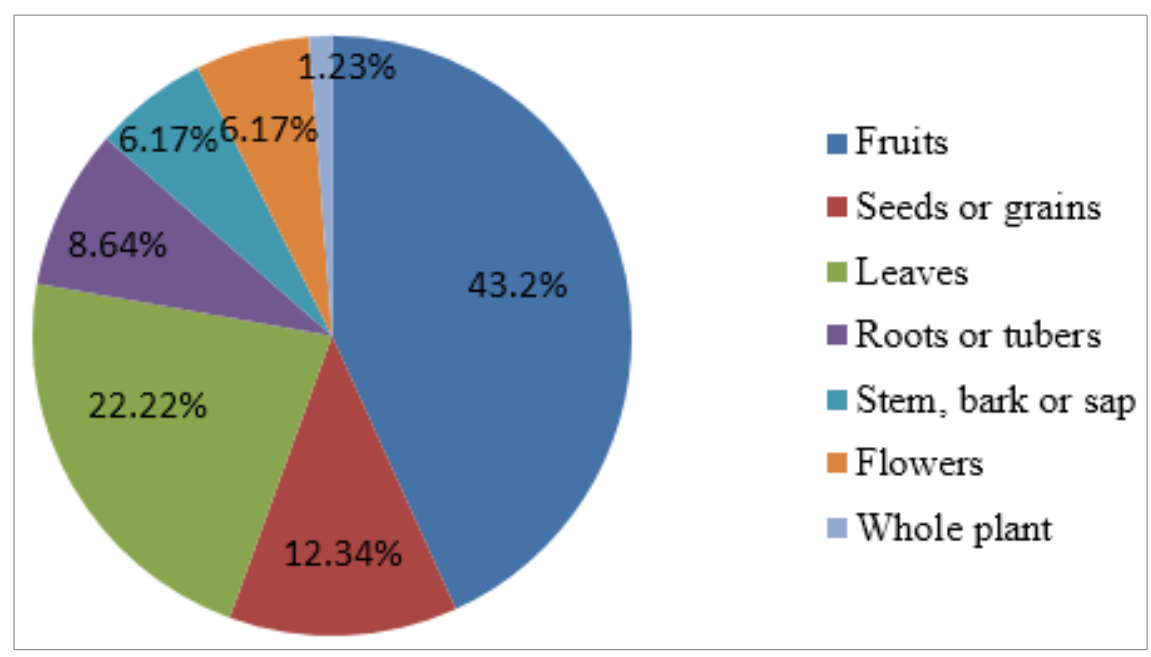

Figure 3. Classification on the basis of plant parts used

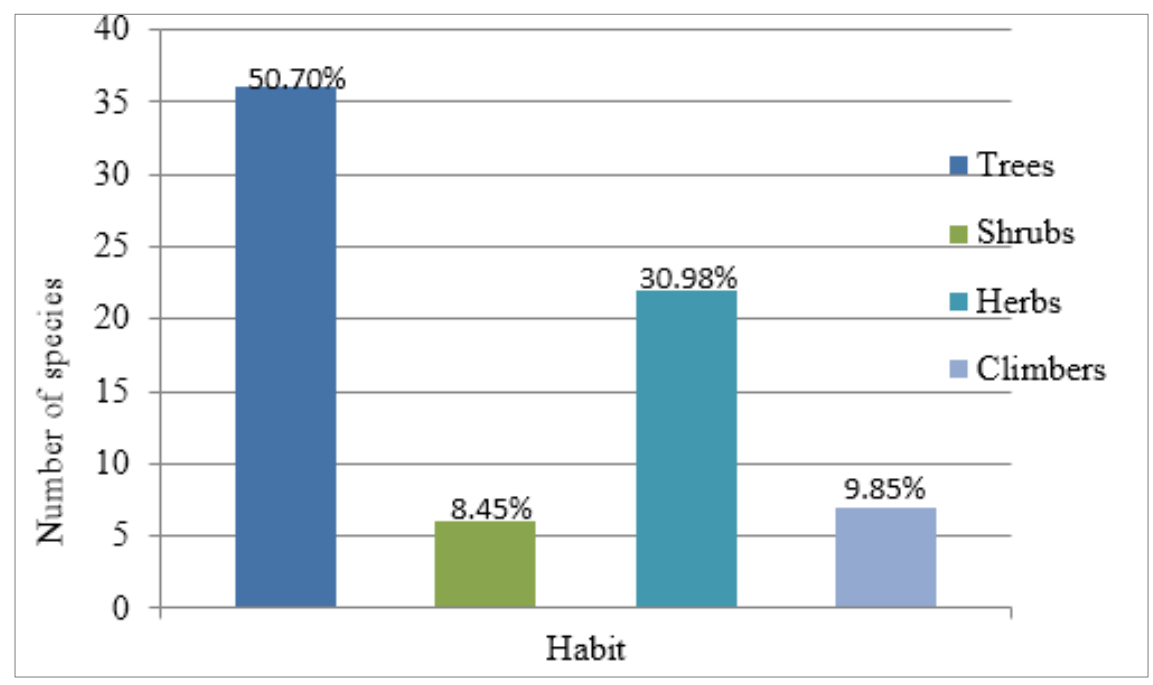

Figure 4. Wild edible plant species in different categories 


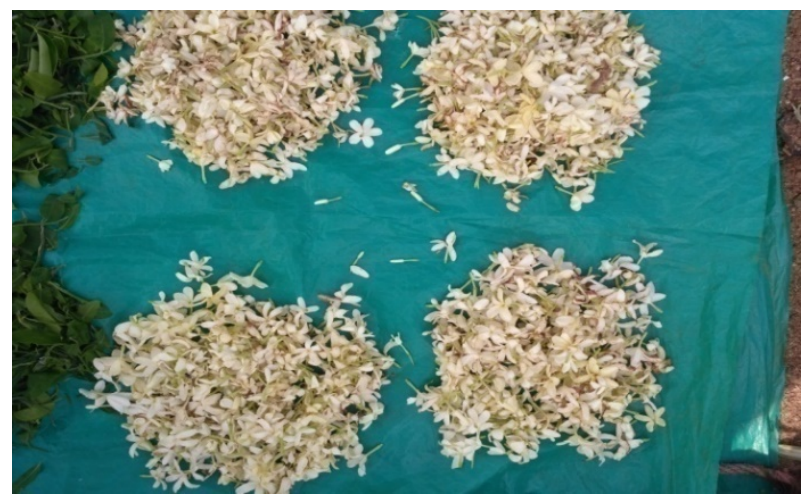

Figure 5. Flowers of Holarrhena pubescens (Buch-Ham.) Wall.ex. G.Don.

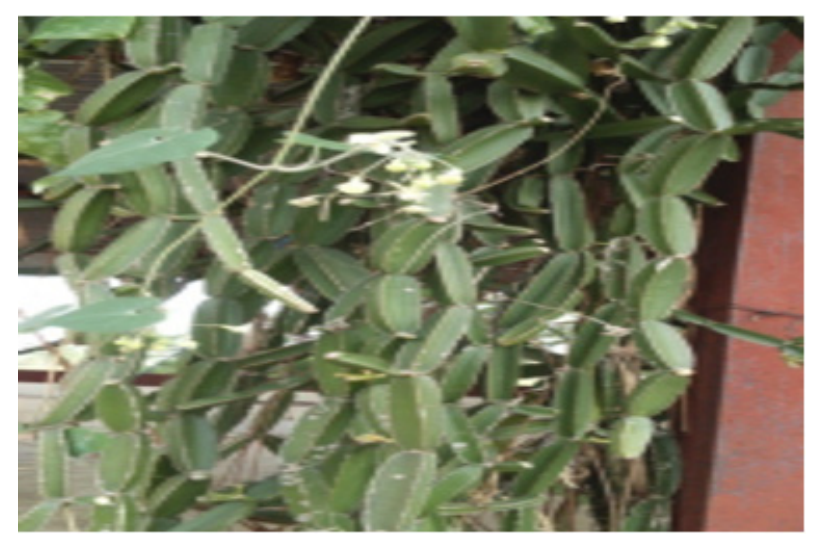

Figure 6. hoots of Cissus quadrangularis L.

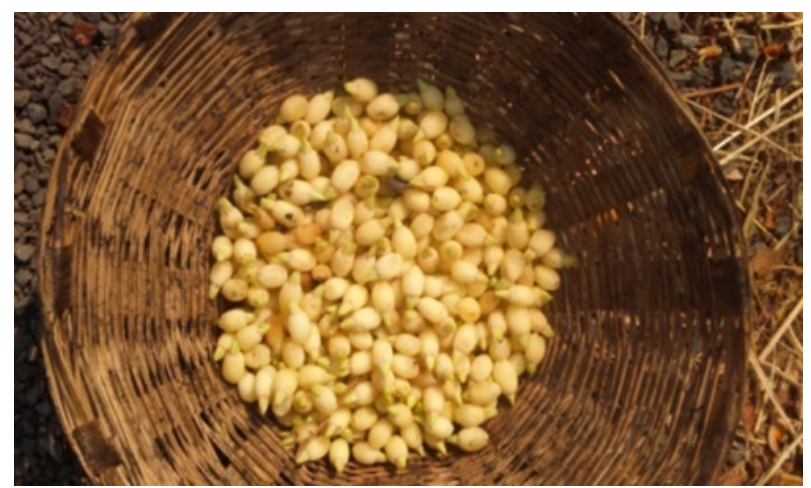

Figure 7. Fleshy flowers of Madhuca longifolia (Koen.) Mac. Var.

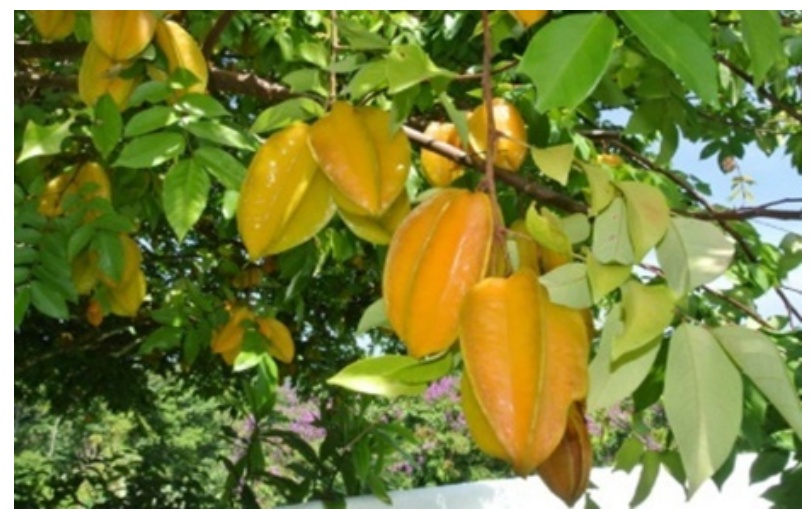

Figure 8. Fruits of Averrhoa carambola L.

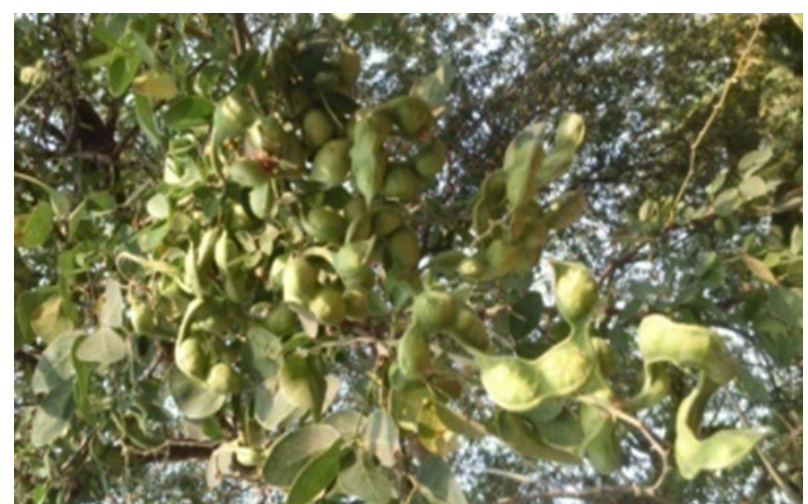

Figure 9. Fruits of Pithecellobium dulce (Roxb.) Benth

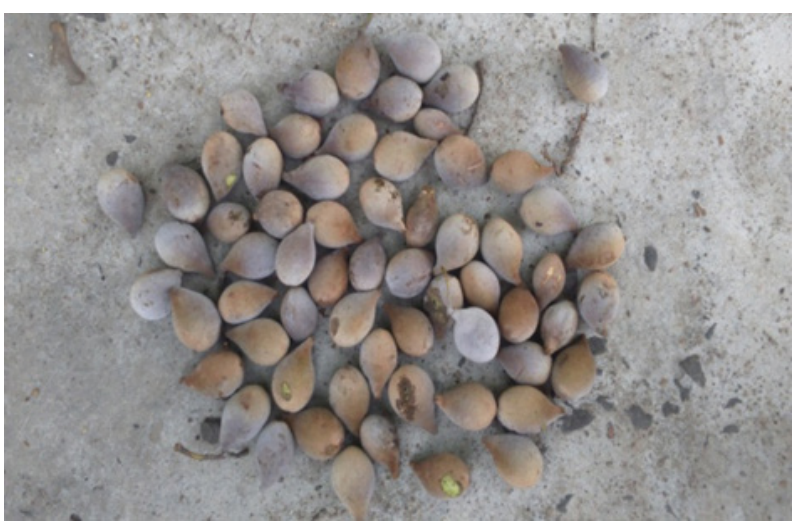

Figure 10. Fruits of Terminalia bellirica Roxb.

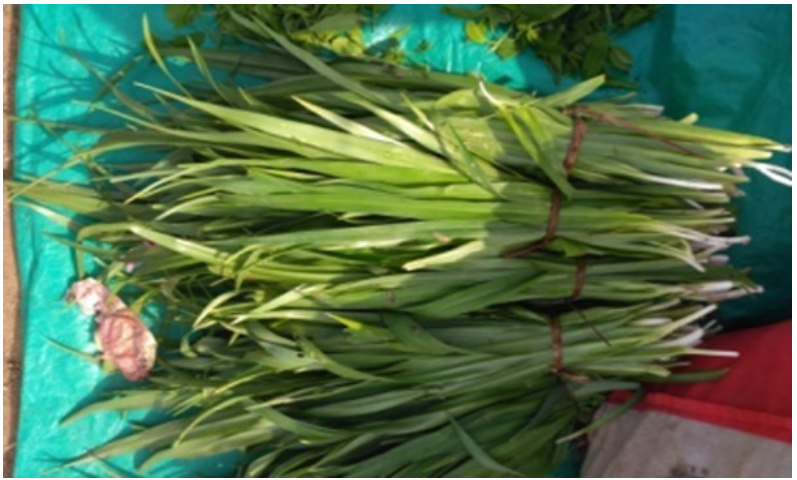

Figure 11. Leafy vegetable of Cryptocoryne retrospiralis (Roxb.) Kunth

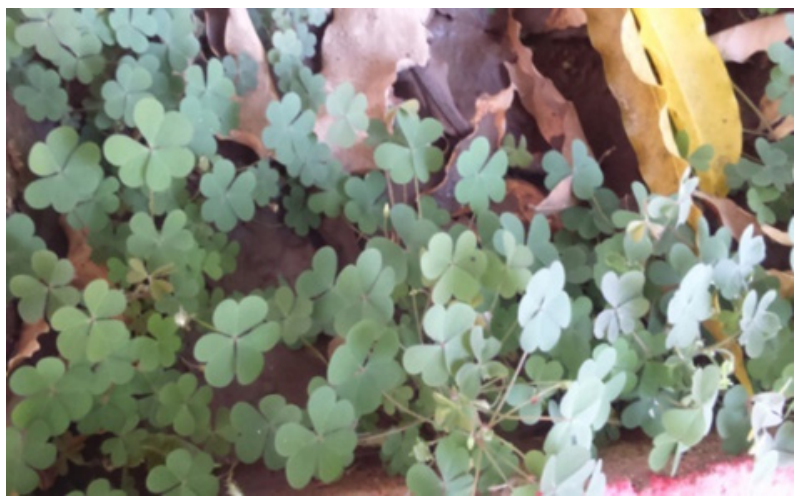

Figure 12. Leafy vegetable of Oxalis corniculata $\mathrm{L}$. 


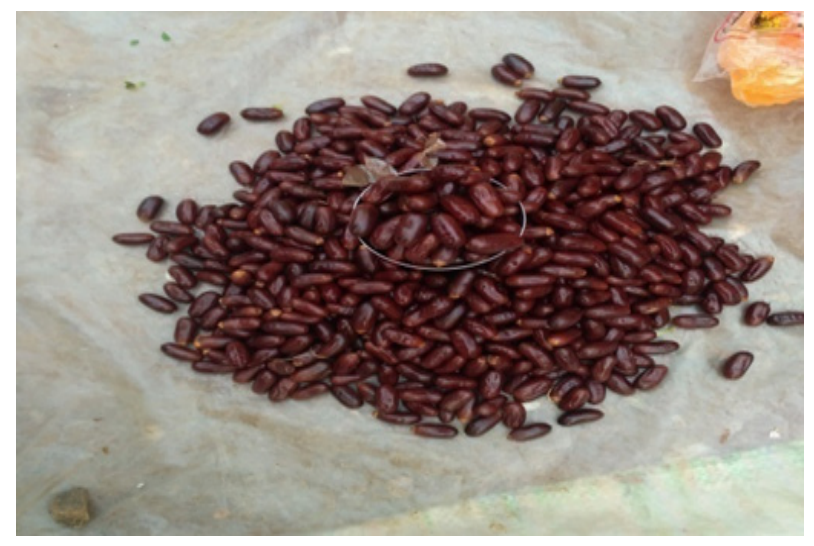

Figure 13. Dried Fruits of Phoenix sylvestris (L.) Roxb.

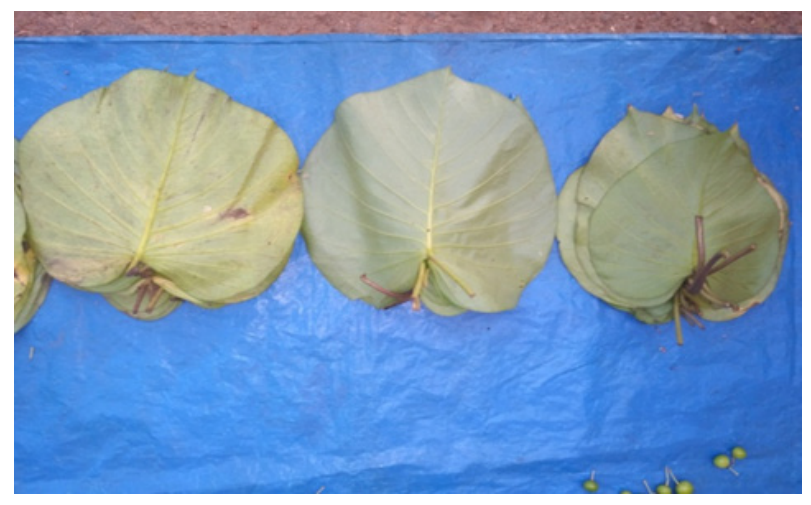

Figure 14. Leafy vegetable of Colocasia esculenta (L.) Schott.

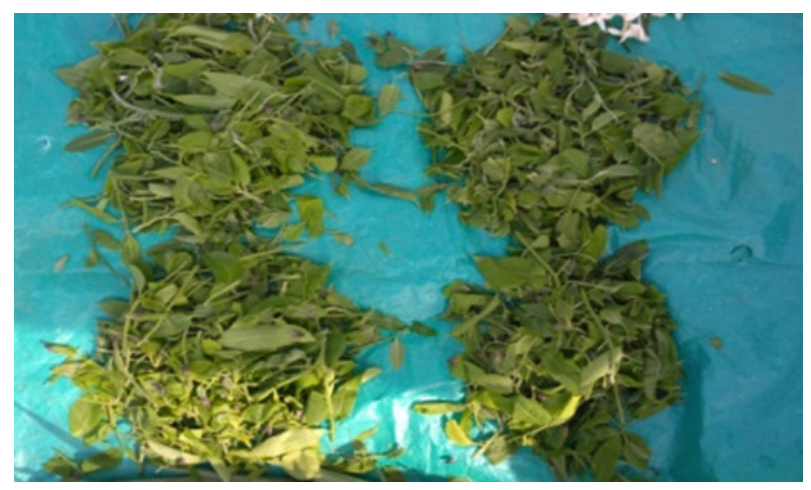

Figure 15. Leafy vegetable of Olax psittacorum (Willd) Vahl.

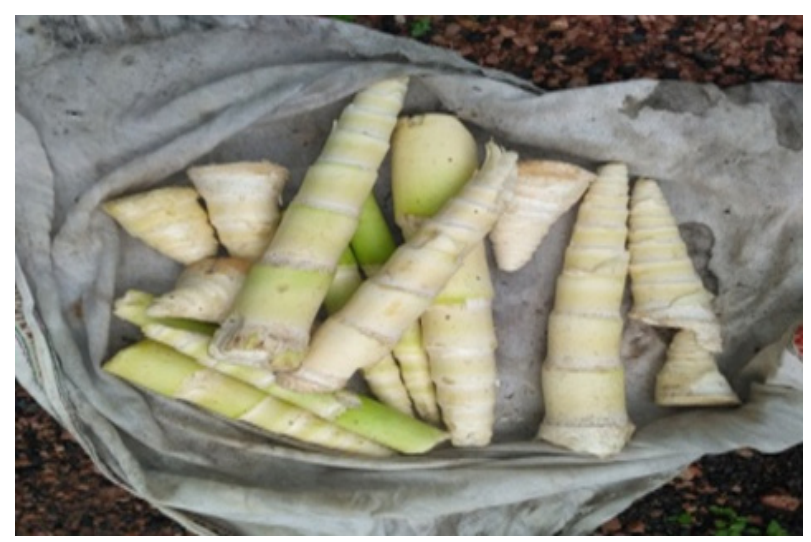

Figure 16. Tender Shoots of Dendrocalamus strictus (Roxb.) Nees.

\section{Results}

Table 2. Family wise distribution of the wild edible plants of Study Area

\begin{tabular}{|c|c|c|c|}
\hline Rank & Family & Genera & Species \\
\hline 1 & Fabaceae & 4 & 4 \\
\hline 2 & Araceae & 4 & 4 \\
\hline 3 & Caesalpiniaceae & 3 & 5 \\
\hline 4 & Poaceae & 3 & 3 \\
\hline 5 & Anacardiaceae & 3 & 3 \\
\hline 6 & Euphorbiaceae & 3 & 3 \\
\hline 7 & Mimosaceae & 2 & 3 \\
\hline 8 & Amaranthaceae & 2 & 3 \\
\hline 9 & Malvaceae & 2 & 2 \\
\hline 10 & Rutaceae & 2 & 2 \\
\hline 11 & Moraceae & 2 & 2 \\
\hline 12 & Oxalidaceae & 2 & 2 \\
\hline 13 & Sapindaceae & 2 & 2 \\
\hline 14 & Verbenaceae & 2 & 2 \\
\hline 15 & Solanaceae & 2 & 2 \\
\hline 16 & Sapotaceae & 2 & 2 \\
\hline 17 & Annonaceae & 1 & 2 \\
\hline 18 & Nymphaeaceae & 1 & 2 \\
\hline 19 & Rhamnaceae & 1 & 2 \\
\hline 20 & Tiliaceae & 1 & 2 \\
\hline 21 & Dioscoreaceae & 1 & 1 \\
\hline 22 & Ebenaceae & 1 & 1 \\
\hline 23 & Rubiaceae & 1 & 1 \\
\hline 24 & Chenopodiaceae & 1 & 1 \\
\hline 25 & Vitaceae & 1 & 1 \\
\hline 26 & Commelinaceae & 1 & 1 \\
\hline 27 & Zingiberaceae & 1 & 1 \\
\hline 28 & Meliaceae & 1 & 1 \\
\hline 29 & Scrophulariaceae & 1 & 1 \\
\hline 30 & Periplocaceae & 1 & 1 \\
\hline 31 & Apocynaceae & 1 & 1 \\
\hline 32 & Convolvulaceae & 1 & 1 \\
\hline 33 & Cucurbitaceae & 1 & 1 \\
\hline 34 & Moringaceae & 1 & 1 \\
\hline 35 & Nelumbonaceae & 1 & 1 \\
\hline 36 & Myrtaceae & 1 & 1 \\
\hline 37 & Combretaceae & 1 & 1 \\
\hline 38 & Trapaceae & 1 & 1 \\
\hline 39 & Olacaceae & 1 & 1 \\
\hline
\end{tabular}

In the present study there are around 71 species of angiosperms belonging to 63 genera and 39 families have been recorded (Table.1, Figures 5-16). Among the 39- 
families, the most widely utilized plant species belong to Caesalpiniaceae (5), Fabaceae (4), Araceae (4), followed by Poaceae (3), Anacardiaceae (3), Euphorbiaceae (3), Mimosaceae (3), Amaranthaceae (3). Malvaceae, Rutaceae, Moraceae, Oxalidaceae, Sapindaceae, Verbenaceae, Solanaceae, Sapotaceae, Annonaceae, Nymphaeaceae, Rhamnaceae, Tiliaceae are with two species each, and the rest of the nineteen families with single species (Table.2). Among the 71 species, $43.2 \%$ of plant species contribute as vegetables by their fruit, $22.22 \%$ by leaf, $12.34 \%$ by seed or grain, $8.64 \%$ by root or tuber, $6.17 \%$ by stem, bark or sap, $6.17 \%$ by flower and $1.23 \%$ by whole plant (Fig.3). While analyzing the life forms of the wild edible vegetable species, it was noticed that $36(50.70 \%)$ species were trees, $22(30.98 \%)$ herbs, $07(9.85 \%)$ climbers and the remaining 06 (8.45\%) were shrubs (Fig.4).

It is evident from the Fig. 2 that most of the wild edible species are used as vegetable/chutney $(49.33 \%)$, followed by raw/ripe fruits(45.33\%), pickles(4\%), roasted seeds(1.33\%).

This study reveals that tribal and rural people living in particular area depend on wild edible plants and have rich knowledge of their utilization.

\section{Discussion}

Many rural parts of the world depend on wild edible plants for their food.[13] Several attempts have been made to list out the wild edibles of Vidharbha region of Maharashtra State. [2,3,14]

Vegetable contain large quantity of vitamin C, Vit. A and Vit. B complex as well as good amount of dietary fibers and phytochemicals. The contents of the wild vegetables also protect our body against various malnutrition, and nutrient disorders that is why they are called as protective food. [15] Such unconventional wild edible plants are sources of proteins, fats, rich source of micro-nutrients and trace elements. $[16,17,18]$

Critical evaluation of the literature reveals that the adequate vegetable consumption can be protective for some chronic diseases such as cancer, obesity, diabetes, cardiovascular diseases, metabolic syndrome, as well as improve risk factors related with these diseases. [19]

In the present study many wild vegetables are also being consumed for various medicinal purposes. Azadirachta indica, Butea monosperma, Syzygium cumini were commonly used to treat deadly disease diabetes. [20] Cassia fistula, Acacia nilotica and Mangifera indica are also used as medicinal plants to cure various women related problem like menstrual disorder, Urinary problems and Leucorrhoea. ${ }^{[21]}$ The tribal people and other villagers residing study area also uses Cassia tora, Dendrocalamus strictus, Grewia tillifolia, Hemidesmus indicus, Semicarpus anacardium for the treatment of various diseases. [22]
Ethnobotanical surveys of wild plants indicate that more than 7000 species have been used for human food at some stage in human history. [23] But this important knowledge is slowly diminishing day by day due to invasion of alien cultures. Documentation of wild edible plants from ethnobotanical approach is important for enhancing the understanding of indigenous knowledge system. [24-26]

\section{Conclusions}

Demand of food and vegetables of increasing population cannot be fulfilled unless we cannot find out the ways and means to increase the production of vegetables and other substitute like wild plants. So it is of the immense need to document the indigenous knowledge of wild edibles for future generations and to encourage the peoples for cultivation of wild edible plants in their home gardens. Further research on cultivation and utilization of wild vegetables would help the tribal and rural people to have better nutrition.

\section{Acknowledgements}

I am very grateful to ethnic people and forest dwellers from the study area for their valuable information that helped me to prepare this Research Article.

\section{REFERENCES}

[1] Pawar, S. And Patil, DA. Ethnobotany of Jalgaon District, Maharashtra. Daya Pulishing House, Delhi, India, pp.1-3,2008.

[2] Bhogaonkar, PY., Marathe, VR. And Kshirsagar, PP. Documentation of Wild Edible Plants of Melghat Forest, Dist. Amravati, Maharashtra State, India. Ethnobotanical Leaflets 14: 751-758,2010.

[3] Setiya, AV., Narkhede, SD. And Dongarwar NM. Exploration and documentation of some wild edible plants used by the aboriginals from Gadchiroli District (M.S.) India. International Advanced Research Journal in Science, Engineering and Technology.3(7) 24-35,2016.

[4] Jain, A.K. And Tiwari, P. Nutritional value of some traditional edible plants used by tribal communities during emergency with reference to Central India. Ind. J. Trad. Knowl. 111, 51-57, 2012.

[5] Deb, D., Sarkar, A., Barma, BD., Datta, BK. And Majumdar, K. Wild Edible Plants and Their Utilization in Traditional Recipes of Tripura, Northeast India. Advances in Biological Research 7 (5): 203-211, 2013.

[6] Choubey, TK. The Working Plan of Gondia Forest Division (Nagpur Circle) Volume - I and II. Government of Maharashtra.2013-14 to 2022-23. 
[7] Jain, SK. Methods and approaches in Ethnobotany. Society of Ethnobotanists, Luknow, India,1989.

[8] Cook, T. Flora of the Presidency of Bombay Vol. I-III. Botanical Survey of India -REPR, Calcutta, India.1958.

[9] Sharma, BD., Karthikeyan, S. And Singh, NP. Flora of Maharashtra State: Monocotyledons. Botanical Survey of India. Calcutta, India, 1996.

[10] Singh, NP. And Karthikeyan, S. Flora of Maharashtra State: Dicotyledons Vol.-I. Botanical Survey of India, Calcutta, India, 2000.

[11] Singh, NP. And Karthikeyan, S. Flora of Maharashtra State: Dicotyledons Vol.-II. Botanical Survey of India, Calcutta, India, 2001.

[12] Ugemuge, NR. Flora of Nagpur District. Shree Prakashan, Nagpur, India, 1986.

[13] Sundriyal, M., Sundriyal, RC. And Sharma, E. Dietary Use of Wild Plant Resources in the Sikkim Himalaya, India. Economic Botany 58(4):626-638, 2003.

[14] Reddy, BM. Wild edible plants of Chandrapur district, Maharashtra, India. Indian Journal of Natural Product and Resources. 3(1),110-117, 2012.

[15] Rai, M., Singh, J. And Pandey, AK. Vegetables: A source of nutritional security. Indian Hort.48(4):14-17, 2004.

[16] Nilegaonkar, S., Vartak, VD. And Chitre, RG. Nutritional evaluation of some wild food plants from Pune and neighbouring districts, Maharashtra state-part-I. Journal of Economic and Taxonomic Botany. 6(3): 629-635,1985.

[17] Kulkarni, DK. Role of ethno-botany in Modern Agriculture. National Conference on Bridging Gap between Ancient and Modern Technologies to Increase Agricultural Productivity, Central Arid Zone Research Institute, Jodhpur, Rajasthan, India.104-115,2006.
[18] Kulkarni, DK., Agte, VV. And Kumbhojkar MS. Leafy vegetables consumed by Mahadeokoli tribe in Western Maharashtra with their nutritional potential. Ethnobotany. 15:34-38, 2003Jain, SK. A Manual of Ethnobotany, IInd Edition. Scientific Publishers, Jodhpur, India,1995.

[19] Narasinga Rao, BS., Deosthale, YG. And Pant, KC. Nutritive value of Indian Foods. National institute of nutrition, Indian Council of Medical Research, Hyderabad, India,1989.

[20] Ghoshal, KP. A Study on Indegenous Medicinal Plants from Gondia District, Maharashtra, India. International Journal of Researches in Biosciences, Agriculture and Technology. $245-248,2014$

[21] Qureshi, PS. Notable medicinal plants used by tribals of Tirora tehsil of Gondia district (M.S.), India, to Cure Women related problems. Int. J. of Life Sciences, Special Issue. A2:194-196,2014.

[22] Cherian, KJ. And Ramteke, DD. Ethnomedicinal plant resources from Navegaon National Park based on socioeconomic documentation from Gondia district, Maharashtra state, India. International Journal for Environmental Rehabilitation and Conservation. 1(1)78 82,2010 .

[23] Grivetti, LE. And Ogle, BM. Value of traditional foods in meeting macro- and micronutrient needs: the wild plant connection. Nutr. Res. Rev. 13: 31-46, 2000.

[24] Uprety, Y., Boon, E. And Poudel, RC. Traditional Use of Plant Resources by Bankariya Ethnic Group in Makawanpur district, central Nepal. GRIN Verlag. 60,2008.

[25] Kayang, H. Tribal knowledge on wild edible plants of Meghalaya, Northeast India. Indian J Trad Knowl.6: 177-181,2007.

[26] Panda, T. Preliminary Study of Ethno-Medicinal Plants Used to Cure Different Diseases in Coastal District of Orissa, India. British J Pharmaco Toxicol.1: 67-71, 2010. 